\title{
An analytic model for the stress-induced anisotropy of dry rocks
}

\author{
Boris Gurevich, ${ }^{3}$ Marina Pervukhina, ${ }^{2}$ and Dina Makarynska ${ }^{1}$
}

\begin{abstract}
One of the main causes of azimuthal anisotropy in sedimentary rocks is anisotropy of tectonic stresses in the earth's crust. We have developed an analytic model for seismic anisotropy caused by the application of a small anisotropic stress. We first considered an isotropic linearly elastic medium (porous or nonporous) permeated by a distribution of discontinuities with random (isotropic) orientation (such as randomly oriented compliant grain contacts or cracks). The geometry of individual discontinuities is not specified. Instead, their behavior is defined by a ratio $B$ of the normal to tangential excess compliances. When this isotropic rock is subjected to a small compressive stress (isotropic or anisotropic), the number of cracks along a particular plane is reduced in proportion to the normal
\end{abstract}

stress traction acting on that plane. This effect is modeled using the Sayers-Kachanov noninteractive approximation. The model predicts that such anisotropic crack closure yields elliptical anisotropy, regardless of the value of the compliance ratio $B$. It also predicts the ratio of Thomsen's anisotropy parameters $\varepsilon / \gamma$ as a function of the compliance ratio $B$ and Poisson's ratio of the unstressed rock. A comparison of the model predictions with the results of laboratory measurements indicates a reasonable agreement for moderate magnitudes of uniaxial stress (as high as $30 \mathrm{MPa}$ ). These results can be used for differentiating stress-induced anisotropy from that caused by aligned fractures. Conversely, if the cause of anisotropy is known, then the anisotropy pattern allows one to estimate P-wave anisotropy from S-wave anisotropy.

\section{INTRODUCTION}

One of the main causes of azimuthal anisotropy in sedimentary rocks is the anisotropy of tectonic stresses in the earth's crust. Stresses affect elastic properties of rocks due to the presence of discontinuities such as cracks and compliant grain contacts. Nonhydrostatic stress can cause elastic anisotropy because the effect of a stress field on a discontinuity depends on the orientation of the discontinuity with respect to the stress field. Knowledge of the pattern of stress-induced anisotropy (expressed, for example, by the ratio of anisotropy parameters) can be useful for distinguishing stress from other causes of anisotropy, such as the presence of aligned fractures. Such patterns can also be used to estimate, say, P-wave anisotropy from S-wave anisotropy estimated from S-wave splitting. Here and below, we use the word "crack" to describe compliant grain contacts, which display a multitude of different orientations, whereas the word "fracture" is used to describe larger-scale features, which form aligned patterns. Mathematically, the orientation distribution function is a smooth function for cracks and a discrete function (a linear combination of a small number of delta functions) for fractures. The term discontinuity is used to describe both cracks and fractures.

Some authors have modeled stress-induced anisotropy by assuming the rock to contain a random distribution of penny-shaped cracks, and by considering the variation of this distribution due to applied stress (see, e.g., Nur, 1971; Sayers, 1988). However, penny-shaped-crack geometry might not give an adequate quantitative description of grain contacts in rocks (Sayers and Han, 2002; Angus et al., 2009; Gurevich et al., 2009b). Alternatively, Mavko et al. (1995) and Sayers (2002, 2007) develop modeling approaches that do not restrict the shape of discontinuities but instead infer their parameters from measurements; see also Verdon et al. (2008). These approaches require numerical calculations to obtain an insight into anisotropy patterns.

Manuscript received by the Editor 17 August 2010; revised manuscript received 5 January 2011; published online 23 May 2011.

${ }^{1}$ Curtin University, Department of Exploration Geophysics, Perth, WA, Australia. E-mail: dgmakarynska@ovi.com.

${ }^{2}$ CSIRO Earth Science and Resource Engineering, Australian Resources Research Centre, Kensington, Perth, WA, Australia. E-mail: Marina. Pervukhina@csiro.au.

${ }^{3}$ Curtin University, Department of Exploration Geophysics, Perth, Australia and CSIRO Earth Science and Resource Engineering, Australian Resources Research Centre, Kensington, Perth, Australia. E-mail: B.Gurevich@curtin.edu.au.

(C) 2011 Society of Exploration Geophysicists. All rights reserved. 
To obtain a simpler and more intuitive insight into these patterns, we make some simplifying assumptions that allow us to compute the anisotropy parameters analytically. Our main assumption is that a rock containing an (initially) isotropic distribution of discontinuities is subjected to a small uniaxial stress (or uniaxial strain) so that the result is a weak anisotropy of the discontinuity orientation distribution, and weak elastic anisotropy.

\section{THEORETICAL MODEL}

\section{Compliance tensor of a cracked solid}

We first consider an isotropic elastic medium (porous or nonporous). We then assume that this medium at ambient stress is permeated by a distribution of cracks with random (isotropic) orientation. For instance, in a granular rock, these cracks might represent randomly distributed and randomly oriented compliant grain contacts. The exact geometry of individual cracks is not specified. Instead, the behavior of cracks is defined by a ratio $B$ of the normal $B_{\mathrm{N}}$ to tangential $B_{\mathrm{T}}$ excess crack compliances. All cracks are assumed to be identical; thus $B$ is the same for all cracks.

When this isotropic rock is subjected to a small compressive stress (isotropic or anisotropic), the number of cracks along a particular plane is reduced in proportion to the normal stress traction acting on that plane. In particular, if the stress is a uniaxial compression along the $x$-axis, then the number of cracks normal to the $x$-axis will reduce most, whereas the number of cracks parallel to the $x$-axis will not reduce at all. We modeled this effect using the Sayers-Kachanov (Sayers and Kachanov, 1995) noninteractive approximation. According to Sayers and Kachanov (1995), the compliance tensor $S_{i j k \ell}$ of a rock with a given distribution of linear-slip cracks (Schoenberg, 1980) can be written as

$$
S_{i j k \ell}=S_{i j k \ell}^{0}+\bar{\alpha}_{i j k \ell}+\beta_{i j k \ell}
$$

with

$$
\bar{\alpha}_{i j k \ell}=\frac{1}{4}\left(\delta_{i k} \alpha_{j \ell}+\delta_{i \ell} \alpha_{j k}+\delta_{j k} \alpha_{i \ell}+\delta_{j \ell} \alpha_{i k}\right) .
$$

Here, $S_{i j k \ell}^{0}$ is the compliance tensor of the intact rock (no cracks), and $\alpha_{i j}$ and $\beta_{i j k \ell}$ are second- and fourth-rank tensors defined by

$$
\begin{gathered}
\alpha_{i j}=\frac{1}{V} \sum_{r} B_{\mathrm{T}}^{(r)} n_{i}^{(r)} n_{j}^{(r)} A^{(r)}, \\
\beta_{i j k \ell}=\frac{1}{V} \sum_{r}\left(B_{\mathrm{N}}^{(r)}-B_{\mathrm{T}}^{(r)}\right) n_{i}^{(r)} n_{j}^{(r)} n_{k}^{(r)} n_{\ell}^{(r)} A^{(r)},
\end{gathered}
$$

where $B_{\mathrm{N}}^{(r)}$ and $B_{\mathrm{T}}^{(r)}$ are the normal and shear compliances of the $r$ th crack in volume $V, n_{i}^{(r)}$ is the $i$ th component of the normal to the crack, and $A^{(r)}$ is the area of the crack. The expression $B_{\mathrm{N}}^{(r)}$ characterizes the normal displacement jump across the crack produced by a unit normal traction, and $B_{\mathrm{T}}^{(r)}$ characterizes the shear displacement jump produced by a unit shear traction. The cracks are assumed to be rotationally symmetrical; that is, $B_{\mathrm{T}}^{(r)}$ is assumed to be independent of the direction of the shear traction within the plane of the crack. In equations 1 through 4 , the cumulative effect of many cracks is assumed to be additive. In other words, the interaction between cracks is neglected (the so-called noninteractive approximation, which is valid for a dilute concentration of cracks).

\section{Effect of stress on crack distribution}

To model the closure of cracks due to the application of anisotropic stress, we assume that $B_{\mathrm{N}}^{(r)}$ and $B_{\mathrm{T}}^{(r)}$ are the same for all cracks, whereas the total area $S=\sum A^{(r)}$ of cracks of a particular orientation (and specific area $s=S / V$ of cracks with that orientation) varies with the direction of the crack normal, and is an exponential function of the normal stress acting in that direction,

$$
s=s^{0} \exp \left(\sigma_{\mathrm{n}} / P_{\mathrm{c}}\right)
$$

where $s^{0}$ is the specific area of all of the cracks before the application of anisotropic stress, $\sigma_{\mathrm{n}}=\sigma_{i j} n_{i} n_{j}$ is normal stress traction acting on the crack surface, and $P_{\mathrm{c}}$ is a characteristic crack closing pressure (Schoenberg, 2002; Shapiro, 2003; Shapiro and Kaselow, 2005; Vlastos et al., 2006). We will now assume that normal stresses are small compared to $P_{\mathrm{c}}$. This assumption is equivalent to the assumption of third-order elasticity (Rasolofosaon, 1998; Prioul et al., 2004; Fuck and Tsvankin, 2009) and results in linear variation of elastic compliances with stress. With this assumption, the exponential expression in Equation 5 can be approximated by a linear expression

$$
s=s^{0}\left(1+\sigma_{\mathrm{n}} / P_{\mathrm{c}}\right) .
$$

For uniaxial stress applied along the $x_{1}$-axis, we have

$$
\sigma_{i j}=\sigma_{0} \delta_{i 1} \delta_{j 1}
$$

so that

$$
\sigma_{\mathrm{n}}=\sigma_{0} n_{1}^{2}=\sigma_{0} \cos ^{2} \vartheta
$$

and

$$
s=s^{0}\left(1+b \cos ^{2} \vartheta\right),
$$

where $\vartheta$ is the angle between the crack normal and $x_{1}$-axis, and $b=\sigma_{0} / P_{\mathrm{c}}$ is the normalized stress magnitude.

\section{Evaluation of crack compliance tensors}

To calculate tensors $\alpha_{i j}$ and $\beta_{i j k \ell}$, it is convenient to adopt a spherical coordinate system with the polar axis $x_{i}$ (because both tensors are symmetrical with respect to their indices, we can assume that $i$ is the smallest index) and with transverse angle $\phi$ measured from any axis that is neither $x_{i}$ nor $x_{1}$. Note that this coordinate system will be different for different components of the tensors. Then, tensor $\alpha_{i j}$ can be written as

$$
\begin{gathered}
\alpha_{i j}=\frac{B_{\mathrm{T}}}{4 \pi} \iint s(\vartheta) n_{i} n_{j} d \Omega=\frac{Z_{\mathrm{T} 0}}{4 \pi} \iint\left(1+b n_{1}^{2}\right) n_{i} n_{j} d \Omega \\
i, j=1, \ldots 3
\end{gathered}
$$

where $d \Omega=\sin \theta d \theta d \phi$ is a body angle element and $Z_{\mathrm{T} 0}$ $=s_{0} B_{\mathrm{T}}$. The double integral in equation 10 can be written in spherical coordinates $\theta, \phi$ as 


$$
\begin{aligned}
\alpha_{i j}= & \frac{Z_{\mathrm{T} 0}}{4 \pi} \int_{0}^{2 \pi} \int_{0}^{\pi}\left(1+b \sin ^{2 m} \phi \sin ^{2 m} \theta \cos ^{2-2 m} \theta\right) \\
& \times \cos ^{n} \phi \sin ^{n+1} \theta \cos ^{2-n} \theta d \theta d \phi
\end{aligned}
$$

where $m=0$ if at least one of the indices $i$ or $j$ is 1 and $m=1$ otherwise; $n=0$ if $i=j$ and $n=1$ if $i \neq j$. This gives

$$
\alpha_{11}=Z_{\mathrm{T} 0}\left(\frac{1}{3}+\frac{b}{5}\right), \quad \alpha_{22}=\alpha_{33}=Z_{\mathrm{T} 0}\left(\frac{1}{3}+\frac{b}{15}\right)
$$

and $\alpha_{12}=\alpha_{23}=\alpha_{13}=0$, which means that tensor $\alpha_{i j}$ is diagonal. It follows that

$$
\begin{aligned}
\bar{\alpha}_{1111} & =\alpha_{11}, \quad \bar{\alpha}_{2222}=\bar{\alpha}_{3333}=\alpha_{22}, \text { and } \\
\bar{\alpha}_{i j i j} & =\frac{1}{4}\left(\alpha_{i i}+\alpha_{j j}\right) .
\end{aligned}
$$

for $i \neq j$ (no summation implied). All other components of tensor $\bar{\alpha}$ are zero.

It then follows that in two-index $6 \times 6$ notation $11 \rightarrow 1$, $22 \rightarrow 2,33 \rightarrow 3,23 \rightarrow 4,13 \rightarrow 5,12 \rightarrow 6$, matrix $\bar{\alpha}_{i j}$ is diagonal with the components

$$
\begin{gathered}
\bar{\alpha}_{11}=\alpha_{11}, \bar{\alpha}_{22}=\bar{\alpha}_{33}=\alpha_{22}, \\
\bar{\alpha}_{44}=\alpha_{22}+\alpha_{33}=2 Z_{\mathrm{T} 0}\left(\frac{1}{3}+\frac{b}{15}\right),
\end{gathered}
$$

and

$$
\bar{\alpha}_{55}=\bar{\alpha}_{66}=\alpha_{11}+\alpha_{22}=2 Z_{\mathrm{T} 0}\left(\frac{1}{3}+\frac{2 b}{15}\right)
$$

(see Sayers, 2002). A similar approach can be used to evaluate the fourth-rank tensor $\beta_{i j k \ell}$. For uniaxially stressed material, all components $i \neq j$ or $k \neq \ell$ vanish, and the remaining components can be written as

$$
\begin{aligned}
\beta_{i i j j} & =\beta_{i j}=\frac{B_{\mathrm{N}}-B_{\mathrm{T}}}{4 \pi} \iint s(\vartheta) n_{i}^{2} n_{j}^{2} d \Omega \\
& =\frac{Z_{N 0}-Z_{\mathrm{T} 0}}{4 \pi} \iint\left(1+b n_{1}^{2}\right) n_{i}^{2} n_{j}^{2} d \Omega, i, j=1, \ldots, 3,
\end{aligned}
$$

with $Z_{N 0}=s_{0} B_{\mathrm{N}}$, or

$$
\begin{aligned}
\beta_{i i j j}= & \beta_{i j}=\frac{Z_{N 0}-Z_{\mathrm{T} 0}}{4 \pi} \int_{0}^{2 \pi} \int_{0}^{\pi}\left(1+b \sin ^{2 m} \phi \sin ^{2 m} \theta \cos ^{2-2 m} \theta\right) \\
& \times \cos ^{2 n} \phi \sin ^{2 n+1} \theta \cos ^{4-2 n} \theta d \theta d \phi
\end{aligned}
$$

where in the four-index version $\beta_{i i j j}$ no summation is implied, whereas the two-index $\beta_{i j}$ version refers to $6 \times 6$ notation. The remaining nonvanishing components of the $6 \times 6$ matrix are (Sayers, 2002) $\beta_{44}=\beta_{23}, \beta_{55}=\beta_{66}=4 \beta_{12}=4 \beta_{13}$. The resulting matrix $\beta_{i j}$ can be written as a sum of isotropic and anisotropic parts,

$$
\boldsymbol{\beta}=\left(Z_{\mathrm{N} 0}-Z_{\mathrm{T} 0}\right)\left(\boldsymbol{\beta}^{\mathrm{is}}+\boldsymbol{\beta}^{\mathrm{an}} b\right)
$$

with

$$
\boldsymbol{\beta}^{\text {is }}=\left[\begin{array}{cccccc}
\frac{1}{5} & \frac{1}{15} & \frac{1}{15} & 0 & 0 & 0 \\
\frac{1}{15} & \frac{1}{5} & \frac{1}{15} & 0 & 0 & 0 \\
\frac{1}{15} & \frac{1}{15} & \frac{1}{5} & 0 & 0 & 0 \\
0 & 0 & 0 & \frac{4}{15} & 0 & 0 \\
0 & 0 & 0 & 0 & \frac{4}{15} & 0 \\
0 & 0 & 0 & 0 & 0 & \frac{4}{15}
\end{array}\right]
$$

and

$$
\boldsymbol{\beta}^{\text {an }}=\left[\begin{array}{cccccc}
\frac{1}{7} & \frac{1}{35} & \frac{1}{35} & 0 & 0 & 0 \\
\frac{1}{35} & \frac{1}{35} & \frac{1}{105} & 0 & 0 & 0 \\
\frac{1}{35} & \frac{1}{105} & \frac{1}{35} & 0 & 0 & 0 \\
0 & 0 & 0 & \frac{4}{105} & 0 & 0 \\
0 & 0 & 0 & 0 & \frac{4}{35} & 0 \\
0 & 0 & 0 & 0 & 0 & \frac{4}{35}
\end{array}\right] .
$$

Similarly, for tensor $\bar{\alpha}$,

$$
\bar{\alpha}=Z_{\mathrm{T} 0}\left(\alpha^{\mathrm{is}}+\alpha^{\mathrm{an}} b\right),
$$

where

$$
\boldsymbol{\alpha}^{\mathrm{is}}=\left[\begin{array}{cccccc}
\frac{1}{3} & 0 & 0 & 0 & 0 & 0 \\
0 & \frac{1}{3} & 0 & 0 & 0 & 0 \\
0 & 0 & \frac{1}{3} & 0 & 0 & 0 \\
0 & 0 & 0 & \frac{2}{3} & 0 & 0 \\
0 & 0 & 0 & 0 & \frac{2}{3} & 0 \\
0 & 0 & 0 & 0 & 0 & \frac{2}{3}
\end{array}\right]
$$

and

$$
\boldsymbol{\alpha}^{\mathrm{an}}=\left[\begin{array}{cccccc}
\frac{1}{5} & 0 & 0 & 0 & 0 & 0 \\
0 & \frac{1}{15} & 0 & 0 & 0 & 0 \\
0 & 0 & \frac{1}{15} & 0 & 0 & 0 \\
0 & 0 & 0 & \frac{2}{15} & 0 & 0 \\
0 & 0 & 0 & 0 & \frac{4}{15} & 0 \\
0 & 0 & 0 & 0 & 0 & \frac{4}{15}
\end{array}\right] .
$$

\section{Elasticity tensors and anisotropy parameters}

Then, equation 1 for the overall compliance tensor can be written in the form

$$
\mathbf{S}=\mathbf{S}^{0}+\Delta \mathbf{S}^{\text {is }}+\Delta \mathbf{S}^{\mathrm{an}} b
$$

where

$$
\Delta \mathbf{S}^{\text {is }}=Z_{\mathrm{T} 0} \boldsymbol{\alpha}^{\text {is }}+\left(Z_{\mathrm{N} 0}-Z_{\mathrm{T} 0}\right) \boldsymbol{\beta}^{\text {is }}
$$

is the excess compliance contribution of the original (isotropic) distribution of cracks in the unstressed rock, and

$$
\Delta \mathbf{S}^{\mathrm{an}}=Z_{\mathrm{T} 0} \boldsymbol{\alpha}^{\mathrm{an}}+\left(Z_{\mathrm{N} 0}-Z_{\mathrm{T} 0}\right) \boldsymbol{\beta}^{\mathrm{an}}
$$


is the excess compliance contribution of cracks created (or closed) due to the application of the anisotropic stress. To isolate the effect of anisotropic stress, we rewrite equation 25 in the form

$$
\mathbf{S}=\mathbf{S}^{\mathrm{is}}+\Delta \mathbf{S}^{\mathrm{an}} b,
$$

with $\mathbf{S}^{\text {is }}=\mathbf{S}^{0}+\Delta \mathbf{S}^{\text {is }}$ defining the (isotropic) compliance of the unstressed rock. By taking an inverse of the total compliance, we can obtain the stiffness tensor, which can also be expressed in powers of $b$,

$$
\mathbf{C}=\mathbf{S}^{-1}=\mathbf{C}^{\text {is }}+\Delta \mathbf{C}^{\text {an }} b+\cdots,
$$

where $\mathbf{C}^{\text {is }}=\left(\mathbf{S}^{\text {is }}\right)^{-1}$ is the stiffness tensor of the unstressed rock. From the stiffness tensor, we can estimate the anisotropy parameters (Thomsen, 1986):

$$
\begin{aligned}
& \varepsilon=\frac{c_{33}-c_{11}}{2 c_{11}}, \\
& \gamma=\frac{c_{44}-c_{55}}{2 c_{55}},
\end{aligned}
$$

and

$$
\delta=\frac{\left(c_{13}+c_{55}\right)^{2}-\left(c_{11}-c_{55}\right)^{2}}{2 c_{11}\left(c_{11}-c_{55}\right)} .
$$

Retaining only the leading (linear) terms in the small parameter $b=\sigma_{0} / P_{\mathrm{c}}$, we obtain

$$
\begin{gathered}
\varepsilon=\delta=\frac{2}{105} \frac{(6+2 v) B_{\mathrm{N}}+(1-2 v) B_{\mathrm{T}}}{1-v} \mu s_{0} \sigma_{0} / P_{\mathrm{c}} \\
=\frac{2}{105} \frac{2 v B+6 B-2 v+1}{1-v} \mu Z_{\mathrm{T} 0} \sigma_{0} / P_{\mathrm{c}} \\
\gamma=\frac{1}{105}\left(3 B_{\mathrm{T}}+4 B_{\mathrm{N}}\right) \mu s_{0} \sigma_{0} / P_{\mathrm{c}} \\
=\frac{1}{105}(3+4 B) \mu Z_{\mathrm{T}} 0 \sigma_{0} / P_{\mathrm{c}}
\end{gathered}
$$

where $\mu$ and $v$ are the shear modulus and Poisson's ratio of the unstressed rock (with an isotropic distribution of cracks), and $B=Z_{\mathrm{N} 0} / Z_{\mathrm{T} 0}=B_{\mathrm{N}} / B_{\mathrm{T}}$ is the ratio of normal to tangential compliances of individual cracks. Thus we see that to the first order in the applied stress, expressions for $\varepsilon$ and $\delta$ are identical. This shows that anisotropic crack closure yields elliptical anisotropy, regardless of the value of the compliance ratio $B$. More precisely, we have shown analytically that if the application of small uniaxial stress results in ellipticity $\varepsilon$ and anellipticity $\varepsilon-\delta$, then

$$
\lim _{\varepsilon \rightarrow 0} \frac{\varepsilon-\delta}{\varepsilon}=0
$$

for any values of the compliance ratio $B$ and host rock's Poisson's ratio $v$. Equations 33 and 34 also show that the ratio $\varepsilon / \gamma$ is fully defined by two independent parameters: Poisson's ratio of the unstressed rock and $B=Z_{\mathrm{N} 0} / Z_{\mathrm{T} 0}=B_{\mathrm{N}} / B_{\mathrm{T}}$,

$$
\frac{\varepsilon}{\gamma}=2 \frac{2 v B+6 B-2 v+1}{(1-v)(3+4 B)} .
$$

Figure $1 \mathrm{a}$ and $\mathrm{b}$ shows the anisotropy parameter $\varepsilon$ and ratio $\varepsilon / \gamma$ versus Poisson's ratio $v$ for different values of compliance ratio $B$. One important thing about these plots is that they are not examples: plot $1 \mathrm{~b}$ is completely universal (within the assumptions of the model). Plot 1a is universal subject to a change of vertical scale according to the magnitude of applied stress. Of course, the results are restricted to small stresses and the resulting weak anisotropy.

In these plots, it is assumed that the shear modulus of the unstressed rocks is the same. If we instead assume constant bulk modulus or, say, constant compressional velocity, the dependency on Poisson's ratio would be somewhat different. However, it is clear that the anisotropy parameters depend only mildly on Poisson's ratio in a range typical for dry consolidated rocks (say, 0.1 to 0.3 ).

In Figure 1b, we also show (as circles) the values of the $\varepsilon / \gamma$ ratio for uniaxial stress and uniaxial strain experiments on Berea sandstone, reported by Scott and Abousleiman (2005). These values plot near the $\mathrm{B}=0.4$ line, which is within the typical range for sandstones (Sayers, 2002; Gurevich et al., 2009b). This is despite the fact that the anisotropy reported by these authors is not weak and significantly deviates from ellipticity, particularly for the uniaxial stress experiment. Possible causes of this are rock damage under nonhydrostatic stress (confirmed by strong acoustic emissions) and the resulting heterogeneity of the sample, as well as difficulties in accurately measuring ultrasonic velocities at oblique angles of incidence.

\section{LABORATORY EXAMPLE}

To illustrate the analytic model derived above, we use the laboratory data of Nur and Simmons (1969) on a sample of Barre Granite. This data set was used previously to test the modeling approach of Mavko et al. (1995) and is attractive because velocities were measured for a range of angles to the axis of symmetry, instead of for only $0^{\circ}, 45^{\circ}$, and $90^{\circ}$, as often is the case.

To test our model against the laboratory data of Nur and Simmons (1969), we first need to determine the parameters of the model. Although a number of parameters were involved in deriving the anisotropy model, all of them can be grouped into four independent parameters: bulk and shear moduli of the unstressed rock $K$ and $\mu$, and the ratio of normal to tangential compliance $B$ and stress sensitivity of $\mathrm{P}$-wave anisotropy $Z_{\mathrm{T} 0} / P_{\mathrm{c}}$. It is clear from equations 33 and 34 that these four parameters are sufficient to describe the stress-induced anisotropy taking into account that the Poisson's ratio of the unstressed rock is given by $v=(3 K-2 \mu) /[2(3 K+\mu)]$.

Similarly to the work of Mavko et al. (1995), our approach is to determine the four model parameters from a hydrostatic stress test, and then use our model to predict the velocity variations in a uniaxially stressed rock without any adjustable parameters. Nur and Simmons (1969) did not report any measurements of their sample under hydrostatic stress; however, such a test on a different sample of Barre Granite was performed by Coyner (1984). The four parameters were determined by fitting Shapiro's (2003) stress sensitivity model to the Coyner data, which consist of Pand $\mathrm{S}$-wave velocities measured as functions of hydrostatic (isotropic) confining stress. The fitting procedure is described in 
Gurevich et al. (2009b); see also Angus et al. (2009). The values of the parameters obtained are $K=13.8 \mathrm{GPa}, \mu=18.3 \mathrm{GPa}, B=$ $1.76, P_{\mathrm{c}}=18.2 \mathrm{MPa}$, and $Z_{\mathrm{T} 0}=0.024 \mathrm{GPa}^{-1}$.

The predictions of the stress-induced anisotropy model against the experimental data of Nur and Simmons (1969) are presented in Figures 2 and 3. Figure 2 shows a comparison between predicted and measured $\mathrm{P}$ - and $\mathrm{S}$-wave velocities as functions of uniaxial stress for three directions. Figure 3 shows the corresponding velocities as functions of the angle of incidence for some pressure levels. The predictions follow the trend of the experimental data for uniaxial stresses as high as $30 \mathrm{MPa}$, after which, as expected, the linear approximation breaks down (note that, strictly speaking, the predictions are expected to work only for stresses smaller than $\left.P_{\mathrm{c}}=18.2 \mathrm{MPa}\right)$. The predictions capture the main trends of the experimental data but underesti-

a)

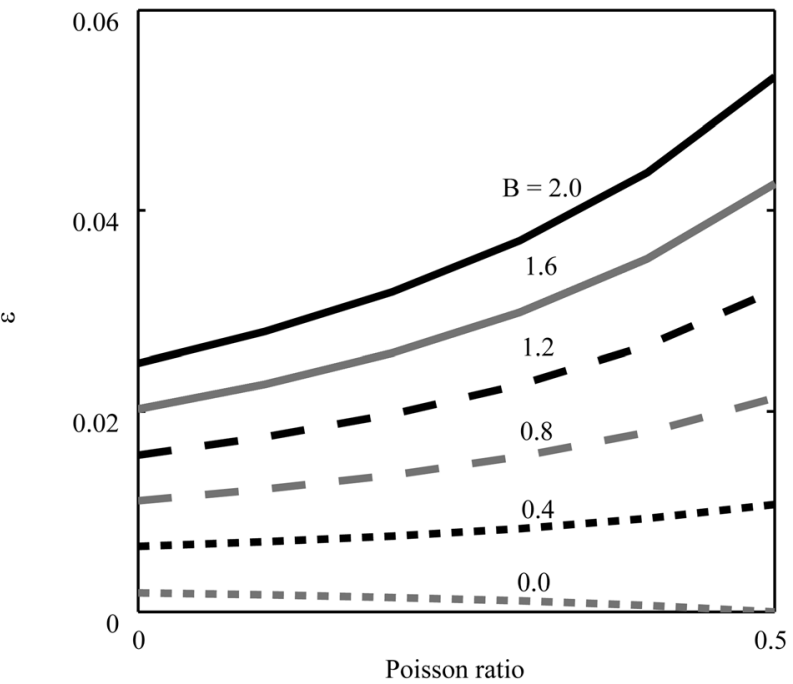

b)

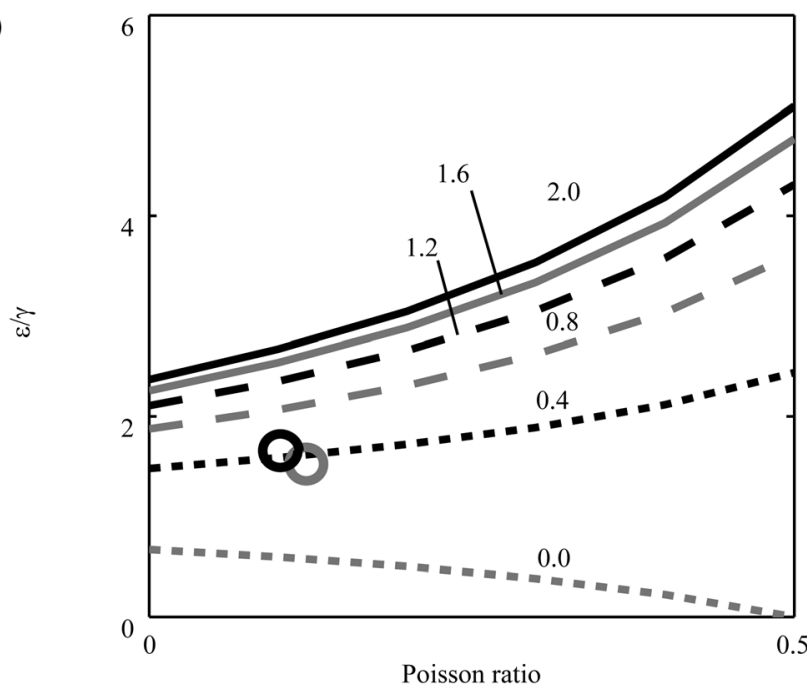

Figure 1. (a) Anisotropy parameter $\varepsilon$, and (b) ratio $\varepsilon / \gamma$, as functions of Poisson's ratio for different values of the compliance ratio $B$. The circles in Figure $1 \mathrm{~b}$ show the values of the $\varepsilon / \gamma$ ratio for uniaxial stress and uniaxial strain experiments on Berea sandstone, respectively, reported by Scott and Abousleiman (2005). mate the magnitude of the P-wave anisotropy, probably due to the opening of cracks parallel to the axis of applied stress, the phenomenon described by Sayers (1988).

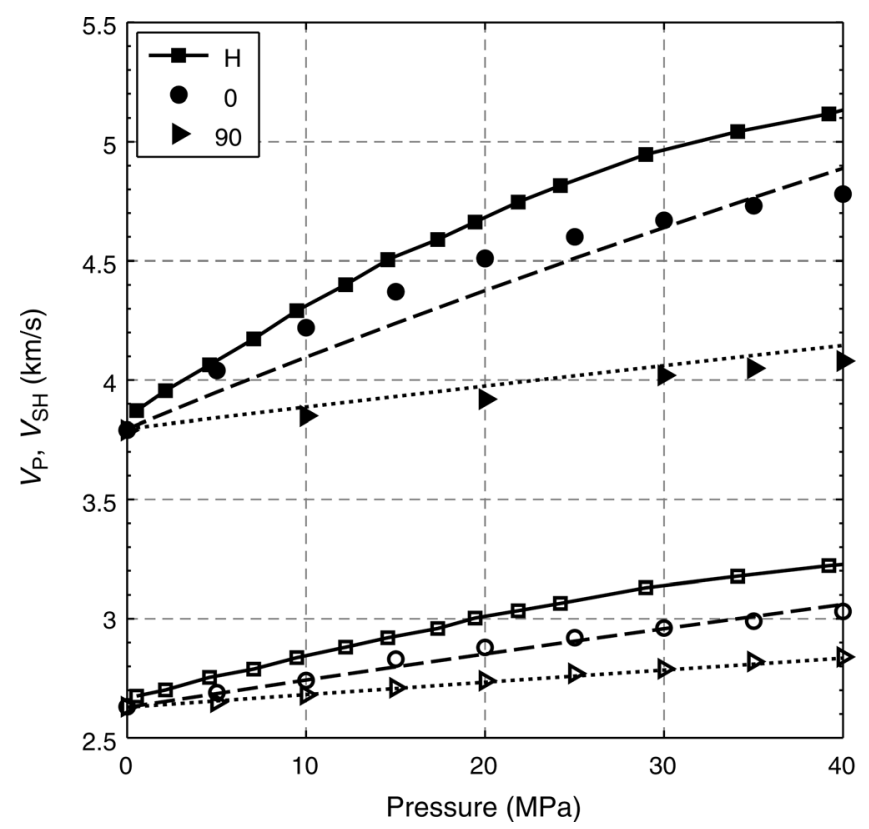

Figure 2. Measured velocities of P-waves (solid symbols) and $\mathrm{SH}-$ waves (open symbols) and model predictions (lines) for a sample of Barre Granite. The squares correspond to the hydrostatic tests of Coyner (1984), which have been used to extract model parameters using the nonlinear fit of Shapiro (2003), indicated by a solid line. The dashed and dotted lines are predictions of the current anisotropy model for uniaxial stress, and the circles and triangles denote the corresponding measurements of Nur and Simmons (1969).

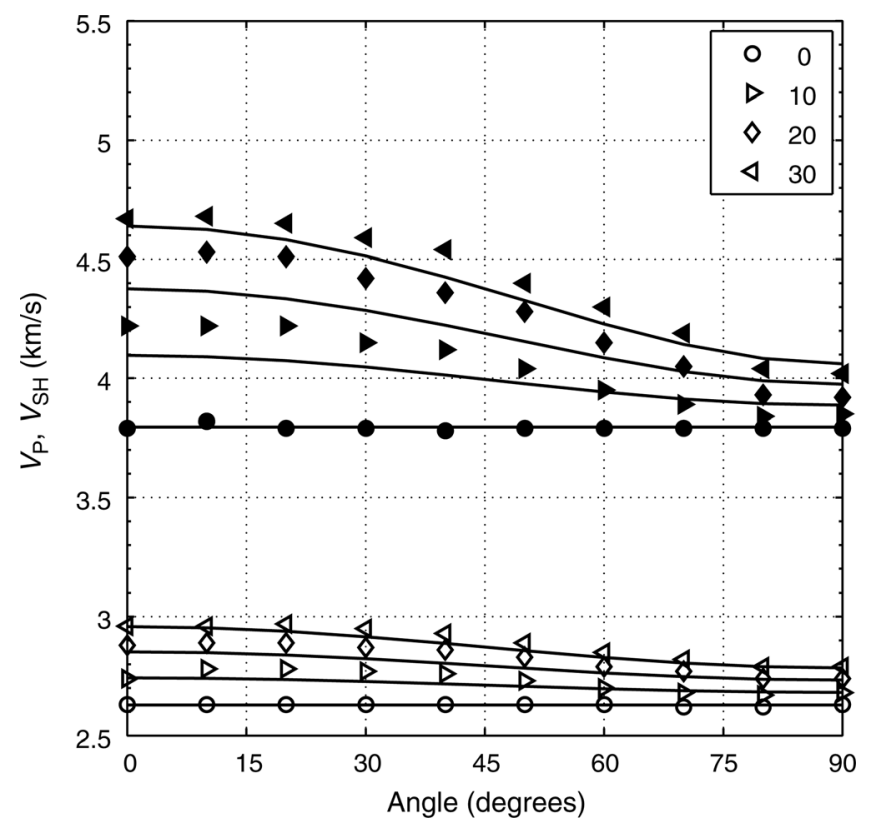

Figure 3. Comparison of angle dependencies of measured velocities of P-waves (solid symbols) and $\mathrm{SH}$-waves (open symbols) and model predictions (lines) for a sample of Barre Granite subjected to different levels of uniaxial stress. 
To explore further the consistency of our model with the measured data, we estimated Thomsen's anisotropy parameters for Barre Granite from angle dependencies of P-, SH-, and SVwave velocities measured by Nur and Simmons (1969). The sample was assumed to be transversely isotropic with a symmetry axis along the direction of uniaxial stress. First, five independent elements of the stiffness matrix were estimated by least-squares fitting of the measured data by exact analytic expressions for angle-dependent phase velocities. The resulting components of the stiffness matrix were then used to compute Thomsen's parameters (equations 30 through 32). These parameters as a function of uniaxial stress are shown in Figure 4. Shown also is the prediction (denoted $\gamma_{p}$ ) of Thomsen's $\gamma$ from $\varepsilon$ obtained by dividing $\varepsilon$ by a constant ratio $\varepsilon / \gamma=2.41$ predicted by our model, equation 36 .

Figure 4 shows that parameters $\varepsilon$ and $\delta$ are quite close for each stress level. This indicates that, in accordance with our model as well as earlier studies, the rock is close to elliptical. Furthermore, the prediction of $\gamma$ from $\varepsilon$ is in good agreement with $\gamma$ values estimated directly from measurements. Note that the crack closing pressure for this rock was estimated to be 18.2 $\mathrm{MPa}$. Even at higher pressures, the predictions of our model are reasonable. Thus it appears that predictions concerning relative magnitudes of anisotropy parameters may hold at higher pressures than the linear stress-dependency model behind those predictions.

\section{ASSUMPTIONS}

The simple model presented in this paper has been designed to provide entirely analytic formulas and physical insight into a problem that until now has been treated mostly numerically.

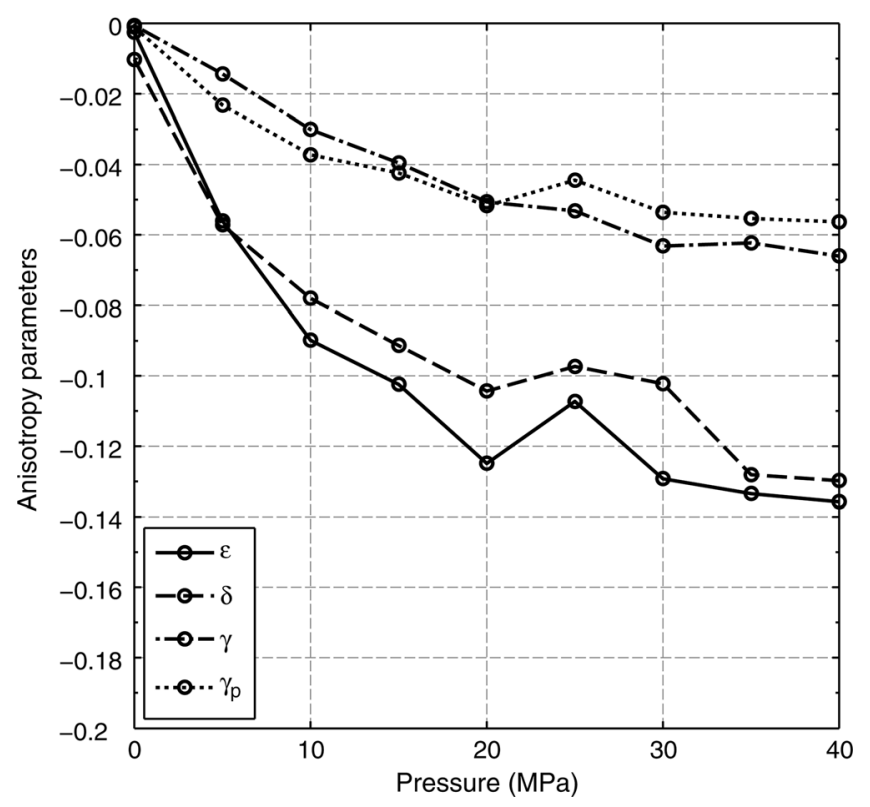

Figure 4. Thomsen's anisotropy parameters $\varepsilon$ (solid line), $\delta$ (dashed line), and $\gamma$ (dashed-dotted line) versus uniaxial stress for Barre Granite as estimated from angle dependencies of $\mathrm{P}-$, $\mathrm{SH}-$, and SV-wave velocities measured by Nur and Simmons (1969). The dotted line shows values of $\gamma$ predicted by dividing $\varepsilon$ by a constant $\varepsilon / \gamma$ ratio predicted by our model (equation 36 ).
This has been achieved at a price of certain simplifying assumptions that have been stated throughout the derivation. Clarity about these assumptions is crucial for understanding the meaning and range of applicability of the results. Thus it will be useful to list these assumptions here and discuss the rationale behind them and their validity.

1) The rock deformation is assumed to be elastic, that is, fully reversible. Any anelastic and irreversible deformations, such as compaction, pressure solution, plasticity, damage, swelling, and hysteresis are excluded. This elasticity assumption is common to many rock physics models, and it is widely used in quantitative modeling and analysis of seismic and acoustic data. It is well known that this assumption does not hold exactly for real rocks. However, for well-consolidated rocks, this assumption usually is considered to be applicable when applied stresses, and, in particular, deviatoric stresses, are not too large, say, below 10 to $30 \mathrm{MPa}$.

2) In an unstressed state, or under hydrostatic stress, all properties of the rock (linear and nonlinear) are assumed to be isotropic. Few, if any, rocks are exactly isotropic, but the goal of this paper is to analyze the anisotropy of rocks due to the application of stress. Thus it is logical to assume that there is no other cause of anisotropy; that is, in the unstressed state the rock is isotropic. In practice, this assumption will apply to rocks whose anisotropy in the unstressed state is much weaker than that caused by the application of uniaxial stress of, say, $20 \mathrm{MPa}$.

3) The rock is assumed to be dry; that is, all cracks and pores (if any) are considered to be empty voids. This assumption appears to be self-explanatory but obviously very restrictive. It is not immediately obvious from our derivation why this assumption is needed. Indeed, it would not be necessary for those rocks wherein all cracks are hydraulically isolated: in such rocks, the fluid saturation of cracks affects only the compliance ratio $B$. However, in nearly all sedimentary and many crystalline rocks, most of the void space is interconnected, and when saturated with fluid this connectivity causes hydraulic interaction between cracks. Because we use the Sayers-Kachanov noninteractive approximation for cracks (see below), we have to exclude hydraulic interaction between them.

In practice, we almost always deal with rocks saturated with some fluid. The question then arises if our model would apply to rocks saturated with, say, air or hydrocarbon gas. This can be answered by quantifying the largest permissible value of the fluid bulk modulus $K_{\mathrm{f}}$. This value can be calculated by assuming that the fluid bulk modulus should be sufficiently small not to cause any noticeable change to the crack stiffness

$$
K_{\mathrm{f}} \ll \alpha \mu,
$$

where $\alpha$ is a characteristic aspect ratio of cracks, usually assumed to be between $10^{-4}$ and $10^{-3}$; and $\mu$ is the shear modulus of the host rock (see, e.g., Hudson, 1981). Although the aspect ratio parameter is not used in our model, it is this parameter that determines the magnitude of fluid effects. For a typical rock with $\mu$ of, say, $10 \mathrm{GPa}$, the fluid modulus should not be larger than $1 \mathrm{MPa}$. It follows that for typical rocks, air at ambient conditions would fulfill condition 37, but a hydrocarbon gas at reservoir pressure might not. 
The results of our model can also be extended to fluid-saturated rocks, as discussed below.

4) Individual grain contacts are assumed to be adequately represented by "infinitely thin" linear-slip cracks (Schoenberg, 1980). This assumption is consistent with the fact that the strain caused by pressures as high as $50 \mathrm{MPa}$ usually is small compared to the relative change of elastic properties. It is thus commonly assumed that all of the stress dependency of rock properties is caused by these compliant pores or cracks at grain-contact areas (see also Shapiro, 2003; Shapiro and Kaselow, 2005; Pervukhina et al., 2010).

5) The cracks representing compliant grain contacts are assumed to be rotationally symmetrical. This does not mean that cracks are circular in shape, but just that on average, all of the cracks aligned parallel to a certain plane are randomly oriented in that plane (see, e.g., Schoenberg and Sayers, 1995).

6) The cracks are assumed to be sufficiently sparse to allow the use of the noninteractive approximation of Sayers and Kachanov (1995). This approximation means that the combined effects of all cracks on the overall rock compliance is a sum of the effects of individual cracks. It has been shown that this approximation is valid for quite significant crack densities so that they can change the elastic moduli of the rock by a factor of 2 (Grechka and Kachanov, 2006). Thus we think this assumption is approximately valid up to pressures that change the moduli by the same factor. Moreover, if parameters such as $B$ and $Z_{\mathrm{T} 0}$ are obtained by inverting experimental data, then these parameters will include crack interactions (see Sayers, 2010).

7) We assume that the number of cracks (or, more generally, the specific surface of cracks) aligned along a particular plane is reduced in proportion to the (compressive) normal stress traction acting on the plane. In other words, the application of stress normal to the crack tends to close that crack. We are not the first to introduce this assumption; see Schoenberg (2002) and Vlastos et al. (2006). This assumption is clearly not universally valid. One effect not covered by this assumption is an increase of the number of cracks parallel to the direction of uniaxial compression (see, e.g., Sayers, 1988; Scott and Abousleiman, 2005). It is still a matter of debate whether such crack opening occurs at all stresses or only above a certain threshold. We think that this is a significant weakness of the model, and we intend to study this effect in the future.

8) The dependence of the number of cracks along a particular plane on normal stress is assumed to be exponential. This assumption, which was based originally on empirical observations, has now found a theoretical justification in the works of Shapiro (2003) and his colleagues.

9) Finally, a crucial simplifying approximation was the linearization of this exponential relationship. This approximation assumes that the applied (static) stress is small compared to the crack closing pressure. This means that the model is limited to stresses that close (or open) only a small portion of all cracks that exist in the unstressed state. By making this assumption, we restrict ourselves to describing only the first (linear) term in the variation of elastic compliances (and, approximately, of the stiffnesses and elastic wave velocities) with stress. This is equivalent to the assumption of third-order elasticity (Rasolofosaon, 1998; Prioul et al., 2004; Fuck and Tsvankin, 2009). In our laboratory example, the model is approximately consistent with the data up to stresses almost twice the closing pressure.

\section{DISCUSSION}

The result concerning the ellipticity of stress-induced anisotropy is consistent with a theoretically proven fact: elastic anisotropy arising from the application of anisotropic stress to an elastic medium that can be described by isotropic third-order elasticity (so that both second- and third-order elasticity tensors are isotropic) is always elliptical (see Rasolofosaon, 1998; Fuck and Tsvankin, 2009). Our model is a particular case of isotropic third-order elasticity. The third order is the result of the linear dependency of the compliance tensor on stress, which is a consequence of the assumption of small stresses (compared to the crack closing pressure). The isotropy of the second-order elasticity tensor is directly assumed (when we say that the unstressed medium is isotropic). The isotropy of the third-order elasticity tensor is the consequence of the isotropic distribution orientation of cracks in the unstressed medium. The prediction of ellipticity at small stresses is illustrated experimentally by Becker et al. (2007).

At the same time, it is known that an isotropic medium permeated by a set of aligned identical fractures is elliptical if, and only if, the fractures are scalar; that is, their compliance ratio $B=Z_{\mathrm{N}} / Z_{\mathrm{T}}$ is 1 . Some experimental and theoretical studies suggest that for real fractures in dry rocks, parameter $B$ is most often significantly smaller than 1 . Typical values for sandstones are between 0.2 and 0.6 (Baltazar et al., 2002; Biwa et al., 2005; Lubbe et al., 2008). This opens up the possibility of differentiating between stress- and fracture-induced azimuthal anisotropy by estimating the degree of anellipticity. Conversely, if the cause of anisotropy is known, then the patterns discussed above allow one to estimate $\mathrm{P}$-wave anisotropy from $\mathrm{S}$-wave anisotropy, by assuming or measuring a value of parameter $B$.

The model presented here is quite similar in concept to the model of Mavko et al. (1995). However, there is one major difference: Mavko et al. (1995) used crack parameters inferred from hydrostatic stress data at each pressure to predict the anisotropy at the same pressure but applied uniaxially. In contrast, we predict the anisotropy using only four parameters for all stress levels. This is achieved by describing the effect of crack closure in both hydrostatic and uniaxial stress tests using a single exponential dependency as proposed by Schoenberg (2002), analytically proven by Shapiro (2003), and verified on experimental data by Pervukhina et al. (2010). The possibility of describing the stress-induced anisotropy by four parameters with clear physical meaning is useful for a number of applications, such as stress estimation from seismic data and the prediction of $\mathrm{P}$ - from S-wave anisotropy derived from shear-wave splitting.

Our model also is consistent with the third-order nonlinear elasticity model of Prioul et al. (2004) and provides physical meaning to its parameters. Similarly to the model of Prioul et al. (2004), our current model predicts only the linear dependency of anisotropy on stress. This is the result of the linearization of the exponential in equation 5. Without this linearization, the integrals in equations 10 and 17 will involve exponentials of trigonometric functions, and will no longer allow analytic 
integration. However, these integrals can be computed numerically (Sayers, 2007).

Our model has been designed for a rock subjected to a small uniaxial stress. However, its prediction of ellipticity is valid also for anisotropy caused by the application of a small uniaxial strain because the state of stress corresponding to a uniaxial strain can be achieved by a superposition of an isotropic stress and a uniaxial stress. On the other hand, the prediction of $\varepsilon / \gamma$ (equation 36; Figure 1b) will be slightly different because it depends on the Poisson's ratio of the medium, which can be affected by the isotropic stress.

The analysis in this paper is valid for cracks in an elastic medium (porous or nonporous) and assumes that interaction between cracks can be neglected. This is a reasonable assumption for dry rocks and for fluid-saturated rocks if the fluid-filled cracks are hydraulically isolated. For interconnected cracks at sufficiently low frequencies, the effect of fluid saturation can be computed using anisotropic Gassmann equations (Gassmann, 1951; Brown and Korringa, 1975; Gurevich, 2003). For higher frequencies, dispersion due to wave-induced flow between cracks and pores may need to be taken into account. Flow between compliant grain contacts and equant pores can be modeled with an anisotropic version of one of the known squirt-flow models (see, e.g., Jones, 1986; Murphy et al., 1986; Chapman et al., 2002; Pride et al., 2004; Gurevich et al., 2010). Flow associated with larger-scale fractures can be modeled using a mesoscopic model based on the theory of poroelasticity (Gurevich et al., 2009a). A detailed analysis of the effect of saturation on anisotropy patterns will be the subject of a separate paper.

\section{CONCLUSIONS}

We have developed an analytic model for seismic anisotropy caused by the application of a small anisotropic stress to an isotropic linearly elastic medium permeated by an isotropic distribution of discontinuities such as randomly oriented compliant grain contacts or cracks. The behavior of cracks is defined by a ratio $B$ of the normal to tangential excess compliances.

The model predicts that such anisotropic crack closure yields elliptical anisotropy, regardless of the value of the compliance ratio $B$. The model also gives an analytic expression for the ratio to Thomsen's anisotropy parameters $\varepsilon / \gamma$ as a function of the compliance ratio $B$ and Poisson's ratio of the unstressed rock. Comparison of the model predictions with the results of a laboratory experiment shows a reasonable agreement for small magnitudes of uniaxial stress (as high as $30 \mathrm{MPa}$ ). These results can be used for differentiating stress-induced anisotropy from that caused by aligned fractures. Conversely, if the cause of seismic anisotropy is known, then the anisotropy pattern allows one to estimate $\mathrm{P}$-wave anisotropy from S-wave anisotropy.

\section{ACKNOWLEDGMENTS}

The work reported in this paper was inspired by numerous discussions with Colin Sayers, particularly at the 2008 SEG Summer Research Workshop on Rock Physics in Galway, Ireland. The authors are also grateful to Serge Shapiro and Andrej Bóna for useful discussions, and to the associate editor and three reviewers for useful advice, which helped improve the paper. BG and DM thank the sponsors of the Curtin Reservoir Geophysics Consortium (CRGC) for financial support.

\section{REFERENCES}

Angus, D. A., J. P. Verdon, O. J. Fisher, and J.-M. Kendall, 2009, Exploring trends in microcrack properties of sedimentary rocks: An audit of dry-core velocity-stress measurements: Geophysics, 74, no. 5, E193E203, doi:10.1190/1.3183940.

Baltazar, A., S. I. Rokhlin, and C. Pecorari, 2002, On the relationship between ultrasonic and micromechanical properties of contacting rough surfaces: Journal of the Mechanics and Physics of Solids, 50, no. 7 , 1397-1416, doi:10.1016/S0022-5096(01)00119-3.

Becker, K., S. A. Shapiro, S. Stanchits, G. Dresen, and S. Vinciguerra, 2007, Stress induced elastic anisotropy of the Etnean basalt: Theoretical and laboratory examination: Geophysical Research Letters, 34, no. 11, L11307, doi:10.1029/2007GL030013.

Biwa, S., A. Suzuki, and N. Ohno, 2005, Evaluation of interface wave velocity, reflection coefficients and interfacial stiffnesses of contacting surfaces: Ultrasonics, 43, no. 6, 495-502, doi:10.1016/j.ultras.2004. 09.003

Brown, R. J. S., and J. Korringa, 1975, On the dependence of the elastic properties of a porous rock on the compressibility of the pore fluid: Geophysics, 40, 608-616, doi:10.1190/1.1440551.

Chapman, M., S. V. Zatsepin, and S. Crampin, 2002, Derivation of a microstructural poroelastic model: Geophysical Journal International, 151, no. 2, 427-451, doi:10.1046/j.1365-246X.2002.01769.x.

Coyner, K. B., 1984, Effects of stress, pore pressure, and pore fluids on bulk strain, velocity, and permeability in rock: Ph.D. thesis, Massachusetts Institute of Technology.

Fuck, R. F., and I. Tsvankin, 2009, Analysis of the symmetry of a stressed medium using nonlinear elasticity: Geophysics, 74, no. 5, WB79WB87, doi:10.1190/1.3157251.

Gassmann, F., 1951, Über die Elastizität poröser Medien: Vierteljahrsschrift der Naturforschenden Gesellschaft in Zürich, 96, 1-23.

Grechka, V., and M. Kachanov, 2006, Effective elasticity of fractured rocks: A snapshot of the work in progress: Geophysics, 71, no. 6, W45W58, doi:10.1190/1.2360212.

Gurevich, B., 2003, Elastic properties of saturated porous rocks with aligned fractures: Journal of Applied Geophysics, 54, no. 3-4, 203-218, doi:10.1016/j.jappgeo.2002.11.002.

Gurevich, B., M. Brajanovski, R. J. Galvin, T. M. Müller, and J. TomsStewart, 2009a, P-wave dispersion and attenuation in fractured and porous reservoirs - Poroelasticity approach: Geophysical Prospecting, 57, no. 2, 225-237, doi:10.1111/j.1365-2478.2009.00785.x.

Gurevich, B., D. Makarynska, O. B. De Paula, and M. Pervukhina, 2010, A simple model for squirt-flow dispersion and attenuation in fluid-saturated granular rocks: Geophysics, 75, no. 6, N109-N120, doi:10.1190/ 1.3509782 .

Gurevich, B., D. Makarynska, and M. Pervukhina, 2009b, Are pennyshaped cracks a good model for compliant porosity?: 79th Annual International Meeting, SEG, Expanded Abstracts, 3431-3434

Hudson, J. A., 1981, Wave speeds and attenuation of elastic waves in material containing cracks: Geophysical Journal of the Royal Astronomical Society, 64, no. 1, 133-150, doi:10.1111/j.1365-246X.1981. tb02662.x.

Jones, T. D., 1986, Pore fluids and frequency-dependent wave propagation in rocks: Geophysics, 51, 1939-1953, doi:10.1190/1.1442050.

Lubbe, R., J. Sothcott, M. H. Worthington, and C. McCann, 2008, Laboratory estimates of normal and shear fracture compliance: Geophysical Prospecting, 56, no. 2, 239-247, doi:10.1111/j.1365-2478.2007. 00688.x.

Mavko, G., T. Mukerji, and N. Godfrey, 1995, Predicting stress-induced velocity anisotropy in rocks: Geophysics, 60, 1081-1087, doi:10.1190/ 1.1443836

Murphy, W. F. I., K. W. Winkler, and R. L. Kleinberg, 1986, Acoustic relaxation in sedimentary rocks: Dependence on grain contacts and fluid saturation: Geophysics, 51, 757-766, doi:10.1190/1.1442128.

Nur, A., 1971, Effects of stress on velocity anisotropy in rocks with cracks: Journal of Geophysical Research, 76, no. 8, 2022-2034.

Nur, A., and G. Simmons, 1969, Stress-induced velocity anisotropy in rock: An experimental study: Journal of Geophysical Research, 74, no. 27, 6667-6674, doi:10.1029/JB074i027p06667.

Pervukhina, M., B. Gurevich, D. N. Dewhurst, and A. F. Siggins, 2010, Applicability of velocity-stress relationship based on the dual porosity concept to isotropic porous rocks: Geophysical Journal International, 181, no. 3, 1473-1479, doi:10.1111/j.1365-246X. 2010.04535.x.

Pride, S. R., J. G. Berryman, and J. M. Harris, 2004, Seismic attenuation due to wave-induced flow: Journal of Geophysical Research, 109, no. B1, B01201, doi:10.1029/2003JB002639.

Prioul, R., A. Bakulin, and V. Bakulin, 2004, Nonlinear rock physics model for estimation of 3D subsurface stress in anisotropic formations: Theory and laboratory verification: Geophysics, 69, 415-425, doi:10.1190/1.1707061 
Rasolofosaon, P., 1998, Stress-induced seismic anisotropy revisited: Revue de l'Institut Français du Pétrole, 53, no. 5, 679-692.

Sayers, C. M., 1988, Stress-induced ultrasonic wave velocity anisotropy in fractured rock: Ultrasonics, 26, no. 6, 311-317, doi:10.1016/0041624X(88)90028-5.

2002, Stress-dependent elastic anisotropy of sandstones: Geophysical Prospecting, 50, no. 1, 85-95, doi:10.1046/j.1365-2478.2002. 00289.x

2007, Effects of borehole stress concentration on elastic wave velocities in sandstones: International Journal of Rock Mechanics and Mining Sciences, 44, no. 7, 1045-1052, doi:10.1016/j.ijrmms. 2007.04.002.

, 2010 , Geophysics under stress: Geomechanical applications of seismic and borehole acoustic waves: SEG Distinguished Instructor Series No. 13.

Sayers, C. M., and D.-H. Han, 2002, The effect of pore fluid on the stressdependent elastic wave velocities in sandstones: 72nd Annual International Meeting, SEG, Expanded Abstracts, 1842-1845.

Sayers, C. M., and M. Kachanov, 1995, Microcrack-induced elastic wave anisotropy of brittle rocks: Journal of Geophysical Research, 100, no. B3, 4149-4156, doi:10.1029/94JB03134.

Schoenberg, M. A., 1980, Elastic wave behavior across linear slip interfaces: Journal of the Acoustical Society of America, 68, no. 5, 15161521, doi:10.1121/1.385077.
_ 2002, Time-dependent anisotropy induced by pore pressure variation in fractured rock: Journal of Seismic Exploration, 11, 83-105.

Schoenberg, M. A., and C. M. Sayers, 1995, Seismic anisotropy of fractured rock: Geophysics, 60, 204-211, doi:10.1190/1.1443748.

Scott, T. E., and Y. Abousleiman, 2005, Acoustic measurements of the anisotropy of dynamic elastic and poromechanics moduli under three stress/strain pathways: Journal of Engineering Mechanics, 131, no. 9, 937-946, doi:10.1061/(ASCE)0733-9399(2005)131:9(937).

Shapiro, S. A., 2003, Elastic piezosensitivity of porous and fractured rocks: Geophysics, 68, 482-486, doi:10.1190/1.1567216.

Shapiro, S. A., and A. Kaselow, 2005, Porosity and elastic anisotropy of rocks under tectonic-stress and pore-pressure changes: Geophysics, 70 , no. 5, N27-N38, doi:10.1190/1.2073884.

Thomsen, L., 1986, Weak elastic anisotropy: Geophysics, 51, 1954-1966 doi: 10.1190/1.1442051.

Verdon, J. P., D. A. Angus, J. M. Kendall, and S. A. Hall, 2008, The effect of microstructure and nonlinear stress on anisotropic seismic velocities: Geophysics, 73, no. 4, D41-D51, doi:10.1190/1.2931680.

Vlastos, S., E. Liu, I. G. Main, M. Schoenberg, C. Narteau, X. Y. Li, and B. Maillot, 2006, Dual simulation of fluid flow and seismic wave propagation in a fractured network: Effects of pore pressure on seismic signature: Geophysical Journal International, 166, no. 2, 825-838, doi:10.1111/j.1365-246X.2006.03060.x. 